\title{
Multicomponent Dipeptide Supramolecular Hydrogels as Fibronectin-Mimetic Biomaterials
}

\author{
Wathsala Liyanage and Bradley L. Nilsson \\ Department of Chemistry, University of Rochester, Rochester, NY, 14627, USA
}

\section{Introduction}

Self-assembled supramolecular peptide hydrogels are important biomaterials that have been designed as matrices for tissue engineering and regenerative medicine [1-4]. Hydrogels derived from low molecular weight dipeptides or functionalized amino acids are a subset of self-assembled peptide hydrogels that have garnered significant recent interest [5-7]. Serious effort has been directed toward engineering low molecular weight hydrogelators that rival peptides in terms of their emergent viscoelastic and biochemical properties. Biochemical cell signaling motifs are often incorporated into supramolecular peptide hydrogels to facilitate cell adhesion, migration, and differentiation. The fibronectin-derived Arg-Gly-Asp (RGD) motif has been frequently incorporated into these types of materials to enable cell adhesion by interacting with integrin receptors.

Herein, we discuss two-component supramolecular hydrogels that functionally bind cell surface integrins in a fibronectin-like manner without the explicit display of the RGD peptide [8]. These hydrogels are composed of Fmoc-3F-Phe-Asp-OH (1) and Fmoc-3F-PheArg- $\mathrm{NH}_{2}$ (2) dipeptides (Figure 1) that coassemble to form a fibril network. These fibrils display Asp and Arg at the surface to spatially mimic the Asp and Arg residues in the RGD motif. NIH 3T3 fibroblast cells adhere to the hydrogel surface via integrin binding in a demonstration of the fibronectin-like biochemical behavior of these materials.

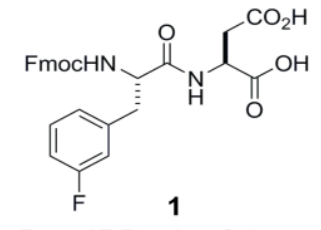

Fmoc-3F-Phe-Asp-OH

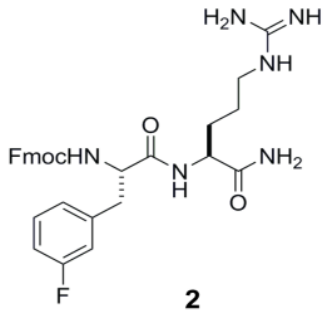

Fmoc-3F-Phe-Arg- $\mathrm{NH}_{2}$

Fig. 1. Structures of Fmoc-3F-Phe-Asp-OH (1) and Fmoc-3F-Phe-Arg- $\mathrm{NH}_{2}(2)$.

\section{Results and Discussion}

The central hypothesis of this study is that supramolecular fibrils that display Asp and Arg in an appropriate spatial approximation of the fibronectin RGD motif will functionally mimic fibronectin in the extracellular matrix and facilitate cell adhesion by binding to integrins. In order to test this hypothesis, we examined coassembly of Fmoc-3F-Phe-Asp-OH (1) and Fmoc-3F-Phe-Arg-NH $\mathrm{N}_{2}$ (2) dipeptides (Figure 1). We have previously found that Fmoc-3F-Phe effectively self-assembles to form self-supporting hydrogels in water [9]. We reasoned that appending either Asp or Arg to the Fmoc-3FPhe assembly motif, giving dipeptides $\mathbf{1}$ and $\mathbf{2}$, would facilitate coassembly of these dipeptides based on complementary charge between the Asp and Arg residues. Further, we anticipated that the resulting fibrils would display these hydrophilic charged residues at the surface where they would be exposed to integrins on cells cultured on these fibrils.

Coassembly and hydrogelation was initiated by dilution of solutions of $\mathbf{1}$ and $\mathbf{2}$ from DMSO into water $\left(9.8 \mathrm{mM}\right.$ combined $\mathbf{1}$ and $\left.\mathbf{2}, 4 \% \mathrm{DMSO} / \mathrm{H}_{2} \mathrm{O} v / v\right)$. We varied the ratio of 2:1 $(1: 1,3: 2,7: 3,4: 1$, $9: 1$ ) in order to assess the hydrogelation capacity of mixtures of these dipeptides. Upon dilution, each of the mixtures initially formed an opaque suspension that became optically transparent, self-supporting hydrogels within 10 minutes. Dipeptide 1 self-assembled to form self-supporting hydrogels, but dipeptide 2 failed to self-assemble independently. The hydrogel forming mixtures were composed of nanotape fibrils with diameters of 10-21 nm. The rheological viscoelasticity of each of the hydrogels was characterized to ensure that the resulting gels would be adequate for cell culture applications. The viscoelasticity of dipeptide hydrogels was measured using dynamic frequency sweep experiments. The gels had storage moduli $\left(\mathrm{G}^{\prime}\right)$ values of $\sim 1400-2500 \mathrm{~Pa}$ and loss moduli $\left(\mathrm{G}^{\prime \prime}\right)$ values of $\sim 20-200 \mathrm{~Pa}$. The hydrogels were sufficiently rigid to support cell culture at the surface of the resulting materials. 
The utility of these hydrogels for cell culture applications was then evaluated [8]. The hydrogels, which were formed in unbuffered water, were perfused by incubation with DMEM in order to remove DMSO from the gels and to prepare them for the introduction of cells [10]. Mouse embryonic fibroblast cells (NIH 3T3) were seeded onto the surface of each coassembled hydrogel $\left(50,000 \mathrm{cells} / \mathrm{cm}^{2}\right)$. As a control, cells were also grown on standard tissue culture plates. After $24 \mathrm{~h}$ of cell seeding, cells incubated on self-assembled hydrogels of dipeptide 1 or 9:1 mixtures of $2: 1$ were found to adopt spherical morphologies indicative of poor cell attachment (Figure 2A and 2B). In contrast, cells grown on 1:1 mixtures of 2:1 (Figure 2D) adopted spindle/polyhedral morphologies identical to those observed on tissue culture plates (Figure 2C), indicative of good cell attachment. The cells remained viable and were shown to continue to proliferate after $>5$ days of incubation on the hydrogel surfaces.
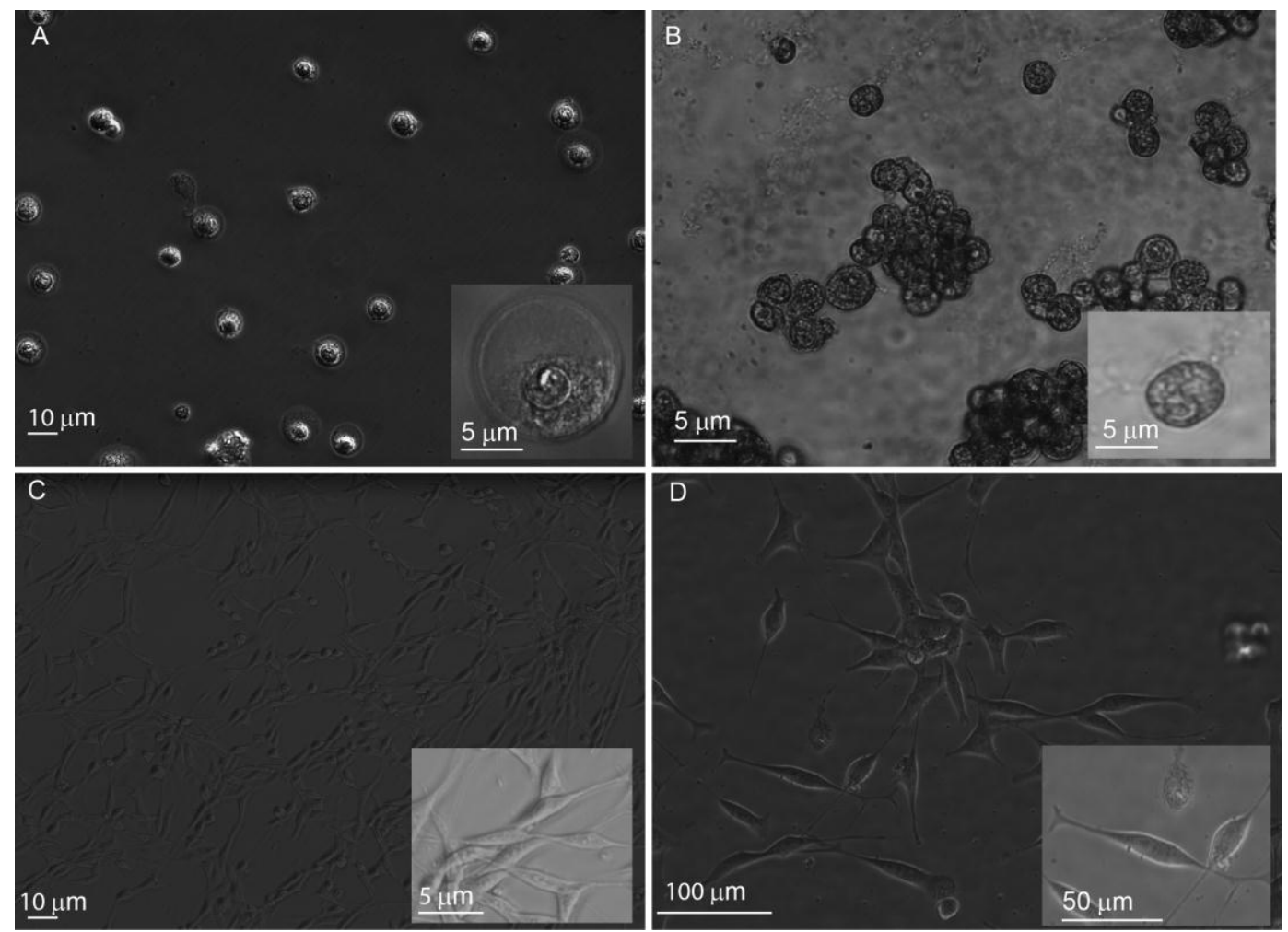

Fig. 2. Images of NIH $3 T 3$ cells seeded on hydrogels of dipeptides 1 and 2 . A. Cells seeded on a selfassembled hydrogel of dipeptide 1 (20x magnification); B. Cells seeded on a 9:1 hydrogel of 2:1 (20x magnification); C. Cells seeded on a tissue culture plate as a control (10x magnification); D. Cells seeded on a 1:1 hydrogel of 2:1 (20x magnification).

The mechanism of cell adhesion to the hydrogels was examined to determine if the gels exhibit fibronectin-like binding to cellular integrins [8]. Integrin-blocking antibodies were used to conduct these cell adhesion analyses (Figure 3). Untreated cells (no integrin blocking) and cells blocked with anti- $\alpha 5$ and $-\beta 1$ integrin antibodies were found to adhere to the $1: 1$ gels of dipeptides $\mathbf{2 : 1}$, while cells blocked with $\alpha \mathrm{v}$ and $\beta 3$ integrin antibodies failed to adhere to the Arg/Asp coassembled nanofiber surfaces and exhibited spherical instead of spindle-like morphologies as shown in Figure 2D. This study suggests that integrin $\alpha v \beta 3$ is responsible for cell adhesion to the Arg/Asp coassembled gels, consistent with many RGD-functionalized materials. Thus, these hydrogels exhibit fibronectinmimetic properties by the noncovalent display of Arg and Asp at the nanofiber surface. 
These studies demonstrate that noncovalent supramolecular display of Arg and Asp provides materials that can effectively mimic the cell adhesive functions of the fibronectin RGD peptide, without covalent connection between the Arg and Asp amino acids. Specifically, we have utilized designed dipeptides (1 and 2, Fmoc-3F-Phe-Asp and Fmoc-3F-Phe-Arg respectively) that are composed of an Fmoc-3F-Phe derivative that functions as an effective assembly motif to arrange the appended Asp and Arg residues at the surface of the resulting fibrils in an alternating fashion. This display of Asp and Arg effectively mimics the spatial arrangement of these residues within covalent RGD peptides. The data indicates that fibrils that have nearly equimolar ratios of Asp and Arg provide hydrogel surfaces that support cell attachment and spreading as well as proliferation. In addition, integrin-blocking assays show that the cells bind to these surfaces in an integrin-dependent manner, similar to RGD-modified materials. These multicomponent coassembled hydrogel materials expand the possibilities for design of novel materials for tissue engineering.

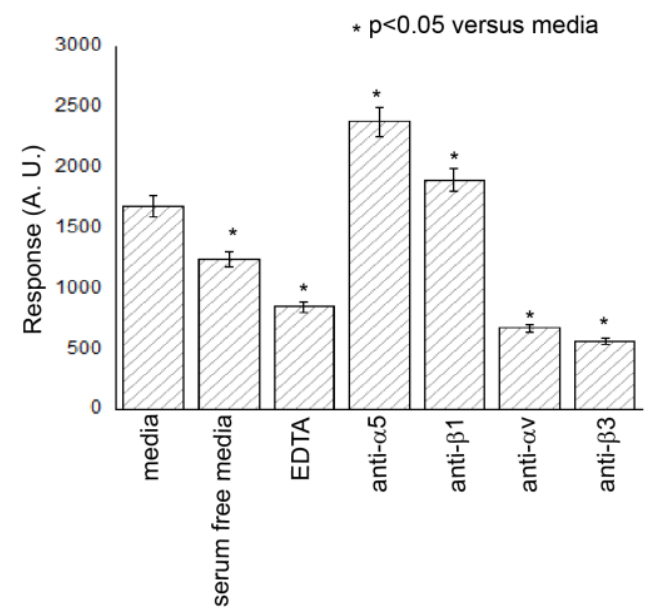

Fig. 3. Integrin-blocking cell adhesion assay. Coassembled hydrogels of 1:1 mixtures of dipeptides $2: 1$ were integrated with integrin-blocking antibodies (anti- $\alpha 5$, anti- $\beta 1$, anti- $\alpha v$, and anti- $\beta 3$ ) [8]. Media, serum-free media, and EDTA controls were conducted on cells incubated in tissue-culture plates. After 2 h of incubation, cells were stained with $2 \mathrm{mM}$ calcein to quantify live cells.

\section{Acknowledgments}

The National Science Foundation supported this research (DMR-1148836). We gratefully acknowledge Karen Bentley (URMC Electron Microscopy Research Core) for her assistance with TEM and SEM imaging and Dr. Scott Kennedy for assistance with CD experiments.

\section{References}

1. Hirst, A.R., Escuder, B., Miravet, J.F., Smith, D.K. Angew. Chem. Int. Ed. Engl. 47, 8002-8018 (2008), http://dx.doi.org/10.1002/anie.200800022

2. Branco, M.C., Schneider, J.P. Acta Biomaterialia 5, 817-831 (2009), http://dx.doi.org/10.1016/j.actbio.2008.09.018

3. Stupp, S.I. Nano Letters 10, 4783-4786 (2010), http://dx.doi.org/10.1021/n1103567y

4. Zhao, X., Pan, F., Xu, H., Yaseen, M., Shan, H., Hauser, C.A.E., Zhang, S., Lu, J.R. Chem. Soc. Rev. 39, 3480-3498 (2010), http://dx.doi.org/10.1039/b915923c

5. Ryan, D.M., Nilsson, B.L. Polym. Chem. 3, 18-33 (2012), http://dx.doi.org/10.1039/c1py00335f

6. Xu, B. Langmuir 25, 8375-8377 (2009), http://dx.doi.org/10.1021/la900987r

7. Fleming, S., Ulijn, R.V. Chem. Soc. Rev. 43, 8150-8177 (2014), http://dx.doi.org/10.1039/c4cs00247d

8. Liyanage, W., Vats, K., Rajbhandary, A., Benoit, D.S.W., Nilsson, B.L. Chem. Commun. 51, 11260-11263 (2015), http://dx.doi.org/10.1039/c5cc03162a

9. Ryan, D.M., Anderson, S.B., Nilsson, B.L. Soft Matter 6, 3220-3231 (2010), http://dx.doi.org/10.1039/c0sm00018c

10. Liyanage, W., Nilsson, B.L., in Lebl, M. (Ed.) Peptides: Peptides Across the Pacific (Proceedings of the 23rd American Peptide Symposium), American Peptide Society, San Diego, 2013, pp. 158-159, http://dx.doi.org/10.17952/23APS.2013/158 